\title{
Neonatal presentation of Caroli's disease
}

\author{
F Keane, N Hadžić, M L Wilkinson, S Qureshi, C Reid, A J Baker, G Mieli-Vergani
}

\begin{abstract}
A neonatal presentation of Caroli's disease with severe cardiac and progressive renal pathology is described. The availability of small paediatric endoscopes ensured early diagnosis. Despite aggressive medical management, the baby died with severe bleeding complications before potentially life saving multiple organ transplantation could take place.

(Arch Dis Child 1997;77:F145-F146)
\end{abstract}

Keywords: Caroli's disease; endoscopic retrograde cholangiopancreatography; pulmonary artery stenosis

Caroli's disease was first described in $1958^{1}$ and is characterised by congenital segmental saccular dilatation of the intrahepatic bile ducts. When hepatic fibrosis occurs, the disease is referred to as Caroli's syndrome. ${ }^{2}$ The mode of inheritance is as yet unknown, but a male prevalence of $75 \%$ has been noted. ${ }^{3}$ Caroli's disease most frequently presents in adolescence with abdominal symptoms and cholangitis. ${ }^{4}$ Associated renal disease ranges from tubular ectasia to infantile polycystic disease. ${ }^{4}$ Recurrent infections, gall stone formation, and development of biliary cirrhosis may severely disrupt the quality of life, ultimately leading to liver transplantation. Caroli's disease has never been reported in infancy.

\section{Case report}

A 5 week old boy presented with persistent conjugated hyperbilirubinaemia, failure to thrive, and pale stools. He was the first child of unrelated Caucasian parents whose medical history was unremarkable. $\mathrm{He}$ was born at 38 weeks of gestation with a birthweight of $3050 \mathrm{~g}$. Labour was induced due to maternal hypertension noted in the final week of pregnancy. There were no febrile illnesses and no medications were given during pregnancy. On the second day of life the baby had a hypoglycaemic episode (blood glucose $1.3 \mathrm{mmol} / \mathrm{l}$ ), which was treated with oral dextrose, but no other early postnatal complications. $\mathrm{He}$ was discharged home breastfed at the age of 7 days, but two weeks later was readmitted on account of prolonged jaundice and poor weight gain. Grossly abnormal prothrombin time (international normalised prothrombin ratio (INR): 10 (normal range 0.8-1.2)), corrected by parenteral vitamin $\mathrm{K}$, prompted referral to our centre.

On admission he was moderately jaundiced and malnourished with no dysmorphic features. A grade $3 / 6$ pansystolic murmur radiating to the axilla was noted. The liver was soft and palpable $1 \mathrm{~cm}$ below the costal margin. There was no splenomegaly or ascites. Laboratory investigations showed a haemoglobin of $10.6 \mathrm{~g} / 1$, a white cell count of $14.6 \times 10^{9} / 1$, a platelet count of $267 \times 10^{9} / 1$, an INR of 1.1 , conjugated bilirubin $124 \mu \mathrm{mol} / 1$ (normal range <20), aspartate aminotransferase 144 IU/1 (normal range 10-50), $\gamma$-glutamyltranspeptidase 1182 IU/1 (normal range 5-50), alkaline phosphatase $186 \mathrm{IU} / 1$ (normal range 30-150), albumin $40 \mathrm{~g} / \mathrm{l}$ (normal range 35-50), urea $0.8 \mathrm{mmol} / 1$ (normal range 2.5-7.5), creatinine $61 \mu \mathrm{mol} / 1$ (normal range 45-120), and cholesterol $2.3 \mathrm{mmol} / \mathrm{l}$ (normal range 3-5).

Extensive work up failed to show any metabolic or other genetic cause of neonatal jaundice. Ophthalmology review showed no posterior embryotoxon. There were no spinal nor vertebral anomalies on $x$-ray pictures. A liver ultrasound scan showed homogeneous parenchyma with a $5 \mathrm{~mm}$ diameter cyst in the superior part of the left lobe. An echocardiogram showed moderately severe pulmonary valve stenosis and stenosis of the right pulmonary artery. The gradient across the pulmonary valve was $55 \mathrm{~mm} \mathrm{Hg}$. A liver biopsy specimen taken at 6 weeks of age showed severe cholestasis with mixed inflammatory infiltrate and ductular proliferation in the portal tracts. Stools were persistently hypocholic and the child remained jaundiced.

A follow up liver biopsy specimen taken two weeks later showed persistent cholestasis, expanded portal tracts with inflammatory infil-

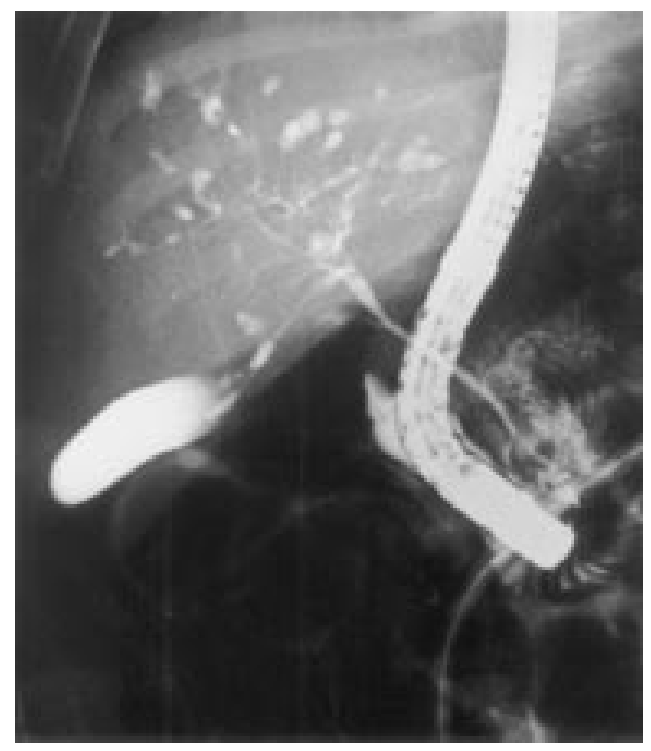

Figure 1 Endoscopic retrograde cholangiopancreatogram taken at 8 weeks of age, showing multisegmental saccular dilatations of intrahepatic bile ducts. 
trate and ductular proliferation, sparse foci of hepatocyte necrosis, giant cell transformation and no fibrosis. Because the biopsy findings were compatible with bile duct obstruction, endoscopic retrograde cholangiopancreatography (ERCP) was performed at the age of 8 weeks. This showed diffuse cystic dilatation of the intrahepatic ducts, diagnostic of Caroli's disease (fig 1). Treatment with fat soluble vitamin supplementation and ursodeoxycholic acid $(15 \mathrm{mg} / \mathrm{kg} / \mathrm{d})$ was started.

At the age of 10 weeks the baby was readmitted with fever and worsening jaundice, suggestive of cholangitis. This time an ultrasound scan showed five small cysts in the liver and enlarged kidneys with no other ultrasonographic abnormalities. Blood cultures grew no pathogens, but he responded clinically to intravenous cefotaxime and amoxycillin.

At 6 months of age nasogastric tube feeding was begun due to severe failure to thrive. Renal function gradually deteriorated (creatinine 78 $\mu \mathrm{mol} / \mathrm{l})$ and two urinary tract infections were documented (Klebsiella, Enterococcus spp). Oral prophylaxis with trimethoprim $(2 \mathrm{mg} / \mathrm{kg} /$ day $)$ was given. Follow up renal ultrasonography showed no cortico-medullary differentiation, but no renal cysts were noted. A micturating cysto-urethrogram showed right sided grade IV vesico-ureteral reflux. A dimethyl-succinic acid scan showed a significant reduction in excretory function of the right kidney. Cardiac catheterisation confirmed right pulmonary artery stenosis with a gradient of $30 \mathrm{~mm} \mathrm{Hg}$ and dysplastic pulmonary valve stenosis. Balloon dilatation of pulmonary valve and right pulmonary artery failed to improve the haemodynamic features.

Two months later repeated episodes of cholangitis, ascites, and poor nutritional state prompted further hospital admissions. Renal function deteriorated rapidly, with creatinine rising to $300 \mu \mathrm{mol} / 1$. The clinical progression of renal failure and the renal ultrasound findings suggested that autosomal recessive polycystic kidney disease, known to be associated with Caroli's disease, was the most likely underlying renal diagnosis. There was worsening vomiting and abdominal distension, and after 48 hours of continuous veno-venous haemodiafiltration, maintenance automated peritoneal dialysis was established. The high risk of bleeding precluded performing a renal biopsy. At 11 months of age the child died after treatment was withdrawn at the parents' request, following severe bleeding complications and subdural haematoma. The parents declined a post mortem examination for their son.

\section{Discussion}

The diagnosis was possible because of the availability of $7.5 \mathrm{~mm}$ paediatric duodenoscope which allows ERCP to be performed safely even in very small infants. ${ }^{5}$ Caroli's disease rather than Caroli's syndrome was diagnosed because of the absence of fibrosis in two liver biopsy specimens..

In the largest series published to date, which included 12 patients with Caroli's syndrome and eight with Caroli's disease, the earliest presentation of the disease was at 3 years of age. $^{3}$ There were no cardiac anomalies reported in either group. Polycystic kidneys were present in $42 \%$ and $25 \%$ of patients with Caroli's syndrome and Caroli's disease, respectively. ${ }^{3}$

Caroli's disease is a result of the remodelling defect of the embryonic ductal plate at the level of large bile ducts. ${ }^{2}$ Recently discovered genetic markers for autosomal recessive polycystic kidney disease may also be involved in the pathogenesis of Caroli's disease. ${ }^{6}$ The clinical course is often complicated by recurrent cholangitis and failure to thrive. The only management option in children with multisegmental Caroli's disease is liver transplantation. ${ }^{4}$ If the disease is restricted to a single lobe, partial hepatectomy may be considered.

Our patient posed a particularly difficult management problem because of associated severe cardiac and progressive renal disease. The only therapeutic prospect would have been a combined liver/kidney and possibly heart transplantation. Unfortunately, the child died before any of these options could be taken.

\footnotetext{
1 Caroli J, Soupault R, Kossakowski J, Plocker L, Paradowska $M$. La dilatation polycystique congenitale des voies biliares M. La dilatation polycystique congenitale des vo

2 Mowat AP. Liver Disorders in Childhood. 3rd Edn. Oxford: Mowat AP. Liver Disorders in Childhood.

3 Summerfield JA, Nagafuchi Y, Sherlock S, Cadafalch J, Scheuer PJ. Hepatobiliary Fibropolycystic diseases: a clinical and histological review of 51 patients. F Hepatol 1986;2:141-56.

4 McEvoy CF, Suchy FJ. Biliary tract disease in children. Pediatr Clin North Am 1996;43:75-98.

5 Wilkinson M, Mieli-Vergani G, Ball C, Portmann B, Mowat AP. Endoscopic retrograde cholangiopancreatography in infantile cholestasis. Arch Dis Child 1991;66:121-3.

6 Mucher G, Wirth B, Zerres K. Refining the map and defining markers of the gene for autosomal recessive polycystic kidney disease on chromosome 6p21.1-p12. Am 尹 Hum Genet 1994;55:1281-4.
} 